\title{
Structural basis of transcription-translation coupling
}

\author{
Chengyuan Wang'*, Vadim Molodtsov*, Emre Firlar ${ }^{2}$, Jason T. Kaelber ${ }^{2}$, Gregor Blaha ${ }^{3}$, Min Su$^{4}+$, \\ Richard H. Ebright ${ }^{1 \dagger}$ \\ ${ }^{1}$ Waksman Institute and Department of Chemistry and Chemical Biology, Rutgers University, Piscataway, NJ 08854, USA. ${ }^{2}$ Rutgers New Jersey CryoEM/CryoET Core \\ Facility and Institute for Quantitative Biomedicine, Rutgers University, Piscataway, NJ 08854, USA. ${ }^{3}$ Department of Biochemistry, University of California, Riverside, CA \\ 92521, USA. ${ }^{4}$ Life Sciences Institute, University of Michigan, Ann Arbor, MI,48109, USA. \\ *These authors contributed equally to this work.
}

†Corresponding author. Email: minsu@umich.edu (M.S.); ebright@waksman.rutgers.edu (R.H.E.)

In bacteria, transcription and translation are coupled processes, in which movement of RNA polymerase (RNAP) synthesizing mRNA is coordinated with movement of the first ribosome translating mRNA. Coupling is modulated by the transcription factors NusG-which is thought to bridge RNAP and ribosomeand NusA. Here, we report cryo-EM structures of Escherichia coli transcription-translation complexes (TTCs) containing different-length mRNA spacers between RNAP and the ribosome active-center P-site. Structures of TTCs containing short spacers show a state incompatible with NusG bridging and NusA binding (TTC-A; previously termed "expressome"). Structures of TTCs containing longer spacers reveal a new state compatible with NusG bridging and NusA binding (TTC-B) and reveal how NusG bridges and NusA binds. We propose that TTC-B mediates NusG- and NusA-dependent transcription-translation coupling.

Bacterial transcription and bacterial translation occur in the same cellular compartment, occur at the same time, and are coordinated processes, in which the rate of transcription by the RNA polymerase (RNAP) molecule synthesizing an mRNA is coordinated with the rate of translation by the first ribosome ("lead ribosome") translating the mRNA [(1-9); see, however, (10)]. Data indicate that the coordination is mediated by transcription elongation factors of the NusG/RfaH family, which contain an $\mathrm{N}$-terminal domain $(\mathrm{N})$ that interacts with RNAP $\beta^{\prime}$ and $\beta$ subunits and a flexibly tethered Cterminal domain $(\mathrm{C})$ that interacts with ribosomal protein S10, and which are thought to bridge, and thereby connect, the RNAP molecule and the lead ribosome (2, 5-9). Further data indicate that the coordination is modulated by the transcription elongation factor NusA (11).

Cramer and co-workers recently reported a $7.6 \AA$ resolution cryo-EM structure of an Escherichia coli transcriptiontranslation complex (TTC) termed the "expressome," obtained by halting a transcription elongation complex (TEC) and allowing a translating ribosome to collide with the halted TEC (12). However, the mRNA molecule in the structure was not fully resolved, precluding determination of the number of mRNA nucleotides between the TEC and the ribosome active center in the structure (12), and the functional relevance of the structure has been challenged, due to its genesis as a collision complex, and due to its incompatibility with simultaneous interaction of NusG-N with RNAP and NusG-C with the ribosome (6-9). Demo et al. recently reported a $\sim 7 \AA$ Å resolution cryo-EM structure of a complex of $E$. coli RNAP and a ribosome $30 \mathrm{~S}$ subunit (13). However, the structure did not contain mRNA, did not position RNAP close to the 30S mRNA-entrance portal, and was incompatible with simultaneous interaction of NusG-N with RNAP and NusG-C with the ribosome (13).

Here, we report cryo-EM structures of E. coli TTCs containing defined-length mRNA spacers between the TEC and the ribosome active-center product site ( $\mathrm{P}$ site), both in the presence of NusG and in the absence of NusG (Fig. 1, figs. S1 to S5, and tables S1 and S2). We prepared synthetic nucleicacid scaffolds that contained (i) DNA and mRNA determinants that direct formation of a TEC upon interaction with RNAP, (ii) an mRNA AUG codon that enables formation of a translation complex having the AUG codon positioned in the ribosome active-center $\mathrm{P}$ site upon interaction with a ribosome and tRNA ${ }^{\mathrm{fMet}}$, and (iii) an mRNA spacer having a length, $n$, of $4,5,6,7,8,9$, or 10 codons (12, 15, 18, 21, 24, 27, or $30 \mathrm{nt})$ between (i) and (ii) (Fig. 1A). We then incubated the nucleicacid scaffolds with RNAP, with ribosome and tRNA ${ }^{\text {fMet }}$, and optionally with NusG and/or NusA, and we determined structures by single-particle-reconstruction cryo-EM (see the materials and methods). With nucleic-acid scaffolds having short spacers ( $\mathrm{n}=4,5,6,7$, or 8$)$, we obtained structures matching the "expressome" of 12 (TTC-A; Figs. 1B, left, and 2; figs. S1 to S3; and table S1). However, with nucleic-acid scaffolds having longer mRNA spacers $(n=8,9$, or 10$)$, we obtained structures of a new molecular assembly with features strongly suggesting it is the molecular assembly that functionally mediates NusG-dependent, NusA-dependent transcription-translation coupling in cells (TTC-B; Figs. 1B, center and right, 3, and 4; figs. S3 to S8; table S1; and movies S1 and S2). 
TTC-A was obtained with nucleic-acid scaffolds having mRNA spacers of $4,5,6,7$, or 8 codons-but not with longer mRNA spacers (Figs. 1B, left, and 2; figs. S1 to S3; and table S1). TTC-A was obtained both in the absence of NusG and in the presence of NusG (figs. S1 to S3 and table S1). EM density maps of 3.7-6.3 $\AA$ resolution were obtained $(\sim 7 \AA$ and $\sim 3.5 \AA$ local resolution for TEC and ribosome, respectively, in best maps), enabling unambiguous rigid-body docking of atomic structures of TEC, ribosome 30S subunit, ribosome 50S subunit with tRNA in active-center P site and exit site (E site), and, where present, NusG-N, followed by manual fitting of residues in the RNAP-ribosome interface and in DNA and mRNA (Figs. 1B, left, and 2; figs. S1 to S3; and table S1).

Remarkably, in TTC-A, the spatial relationship of RNAP relative to the ribosome is identical in structures obtained with nucleic-acid scaffolds having mRNA spacer lengths of 4, 5, 6, 7, and 8 codons (fig. S1F). High-resolution data for TTCA reveal that differences in mRNA spacer length are accommodated through differences in extents of compaction of mRNA in the RNAP RNA-exit channel and RNAP-ribosome interface (Fig. 2B). As mRNA spacer length increases from 4 codons to 5 codons to 6 codons, the number of mRNA nucleotides in the RNAP RNA-exit channel and RNAP-ribosome interface increases from $7 \mathrm{nt}$ ( $5 \mathrm{nt}$ in exit channel; $2 \mathrm{nt}$ in interface) to $10 \mathrm{nt}$ ( $7 \mathrm{nt}$ in exit channel; $3 \mathrm{nt}$ in interface) to $13 \mathrm{nt}$ (9 nt in exit channel; $4 \mathrm{nt}$ in interface) (Fig. 2B, subpanels 1 to 3). When the mRNA spacer length increases to 7 or 8 codons, 16 or 19 nt of mRNA are accommodated in the RNAP RNA-exit channel and RNAP-ribosome interface, and the 16 or $19 \mathrm{nt}$ of mRNA show disorder, indicating they adopt an ensemble of different conformations (Fig. 2B, subpanels 4 and 5). We point out that the volume of the RNAP RNA-exit channel and RNAP-ribosome interface cannot accommodate more than 19 nt of mRNA without changing the conformation of the RNAP RNA-exit channel or disrupting the RNAP-ribosome interface, and we suggest that this accounts for our observations that TTC-A is obtained at relatively low particle populations with a nucleic-acid scaffold having an mRNA spacer length of 8 codons (18\% vs. $91 \%$ for nucleic-acid scaffold having mRNA spacer length of 4 codons; figs. S1 and S3) and is not obtained with nucleic-acid scaffolds having mRNA spacer lengths $>8$ codons (figs. S4 and S5). The mRNA spacers analyzed in this work contained only U (Fig. 1A); because $\mathrm{U}$ is the RNA nucleotide having the smallest volume, the mRNA-spacer-length cut-off of 8 codons observed in this work is likely to represent an upper bound.

In TTC-A, the RNAP-ribosome interface is extensive $(3,742$ $\AA^{2}$ buried surface area) and involves contacts of RNAP $\beta^{\prime}$ zincbinding domain (ZBD), RNAP $\beta$ flap, and RNAP $\alpha^{\mathrm{I}}$ with ribosomal proteins S4, S3, and S10, respectively (Fig. 2, C and D).

In EM density maps of TTC-A, density is absent for RNAP $\omega$ subunit, indicating that RNAP $\omega$ subunit is either absent, or at a low occupancy level, or disordered (Fig. 2E). Molecular modelling suggests that, if RNAP $\omega$ were present and fully folded, the C-terminal $\alpha$-helix of $\omega$ would clash with the ribosome (Fig. 2E).

In EM density maps of TTC-A obtained in the presence of NusG, EM density is present for NusG-N (residues 1-118) at its expected binding location on the RNAP $\beta^{\prime}$ clamp helices and the RNAP $\beta$ pincer tip [(14); Fig. 1B, left, and fig. S1H], but is absent for the NusG linker and NusG-C, consistent with unrestricted motion of the linker and NusG-C relative to NusG-N [(14); Fig. 1B, left]. Density maps for TTC-A obtained in the absence of NusG are identical to those obtained in the presence of NusG, except that density for NusG-N is missing (fig. S2). Model building indicates that the shortest sterically allowed distance between NusG-N bound to RNAP and NusG$\mathrm{C}$ modeled as bound to its molecular target on the ribosome, ribosomal protein $\mathrm{S} 10(2,5-9)$, is $160 \AA$ in TTC-A-which is 1.9 times the maximum length of the NusG linker-indicating that TTC-A is incompatible with NusG bridging of RNAP and S10 (fig. S9A).

Molecular modelling indicates that TTC-A also is incompatible with other known functional properties of transcription elongation, pausing, and termination in E. coli. TTC-A is sterically incompatible with binding of NusA [(15); fig. S10A], formation of a $21 \mathrm{Q}$ antitermination complex [(16, 17); fig. S10B], and formation of pause and termination RNA hairpins $[(15,18,19)$; fig. S10, C and D]. TTC-A also appears to be incompatible with ribosome $30 \mathrm{~S}$-head swivelling, the $21^{\circ}$ rotation of the ribosome $30 \mathrm{~S}$ head relative to the ribosome $30 \mathrm{~S}$ body that occurs during ribosome translocation [(20-22); fig. S11A and movie S3]. The RNAP-ribosome interface in TTC-A spans the $30 \mathrm{~S}$ head and $30 \mathrm{~S}$ body in the unswivelled state (Fig. 2C and fig. S11A, left) and is expected to be disrupted upon swivelling (loss of 1,972 $\mathrm{A}^{2}$ buried surface area; fig. S11A, right). The finding that TTC-A-the "expressome" of 12- lacks RNAP $\omega$ subunit, is incompatible with NusG bridging, and is incompatible with known functional properties of transcription and translation in E. coli indicates that TTC-A is unlikely to be functionally relevant to transcription-translation coupling under most conditions in E. coli. We propose that TTCA is either: (i) a specialized complex that mediates transcription-translation coupling under specialized circumstances (e.g., transcription-translation coupling by RNAP deficient in $\omega$ or by ribosomes inactive in translocation), or (ii) an anomalous complex formed when the mRNA spacer between RNAP and ribosome is anomalously short (e.g., "collisionome" or "crash-ome").

TTC-B was obtained with nucleic-acid scaffolds having mRNA spacer lengths of 8,9 , or 10 codons-but not with shorter mRNA spacers (Figs. 1B, 3, and 4; figs. S3 to S7; and table S1). TTC-B was obtained only when NusG was present 
(figs. S3 to S8 and table S1) and was obtained both without bound NusA and with bound NusA (figs. S3 to S7 and table S1). TTC-B differs from TTC-A by translation of RNAP relative to the ribosome by $\sim 70 \AA$ and rotation of RNAP relative to the ribosome by $\sim 180^{\circ}$ (Fig. 1B and movie S1). EM density maps at 3.1-12.6 $\AA$ resolution were obtained, $(\sim 7 \AA$ and $\sim 3 \AA$ local resolution for TEC and ribosome, respectively, in best maps), enabling unambiguous rigid-body docking of atomic structures of components, followed by manual fitting (Figs. 3 and 4 and figs. S3 to S7). TTC-B is identical to the NusG-bridged complex reported in a preprint by Weixlbaumer and co-workers (23) and is different from the NusA-containing complex reported in a preprint by Mahamid, Rappsilber, and co-workers (24).

In contrast to in TTC-A, where the RNAP RNA-exit channel is coupled directly to the ribosome mRNA entrance portal, in TTC-B, the RNAP RNA-exit channel is separated by $\sim 60$ $\AA$ from the ribosome mRNA entrance portal (Fig. 1B). In TTC$\mathrm{B}, \mathrm{a} \sim 60 \AA, \sim 11 \mathrm{nt}$, mRNA segment connects the RNAP RNAexit channel and the ribosome mRNA entry portal, running along the surface of the ribosome $30 \mathrm{~S}$ head, making favorable electrostatic interactions with positively charged residues in ribosomal protein S3 and RNAP $\beta^{\prime}$ ZBD (Figs. 3B and 4B and figs. S3F and $\mathrm{S} 4 \mathrm{G}$ ). The requirement for this additional $\sim 11 \mathrm{nt}$ mRNA segment accounts for the fact that TTC-B is obtained only with nucleic-acid scaffolds having mRNA spacer lengths $\geq 8$ codons.

In TTC-B, the spatial relationship of RNAP relative to the ribosome is identical in structures obtained with mRNA spacer lengths of 8,9 , and 10 codons (figs. S4F and S5G). Analogously to in TTC-A, in TTC-B, differences in mRNA spacer length are accommodated through differences in extents of compaction of mRNA in the RNAP RNA-exit channel (Figs. 3B and 4B). As mRNA spacer length increases from 8 to 9 to 10 codons, the number of mRNA nucleotides in the RNAP RNA-exit channel increases from $\sim 8 \mathrm{nt}$ to $\sim 11 \mathrm{nt}$ to $\sim 14$ nt (disordered in each case; Figs. 3B and 4B). Assuming that the volume of the RNAP RNA-exit channel allows it to accommodate up to $\sim 15 \mathrm{nt}$ of mRNA (see above), it seems likely that mRNA spacer lengths up to 10 codons could be accommodated in TTC-B. Noting that the mRNA segments in the RNAP-ribosome interface and near ribosomal protein S3 in TTC-B are solvent-accessible, it also seems possible that longer, possibly much longer, mRNA spacer lengths could be accommodated by looping of, or secondary-structure formation in, these mRNA segments.

In TTC-B, the interaction between RNAP and ribosome is small, involving only contact between the RNAP $\beta^{\prime}$ ZBD sequence and ribosomal protein S3 (224 $\AA^{2}$ buried surface area; Figs. 3, C and D, and 4, C to E).

In TTC-B, the RNAP-ribosome interaction is supplemented by bridging of RNAP and the ribosome by NusG, involving simultaneous binding of NusG-N to RNAP and binding of NusG-C to ribosomal protein S10 (1,409 $\AA^{2}$ buried surface area for NusG-C and S10; Figs. 1B, 3, A, C, and D, and 4, A and C to E; and figs. S3G, S4H, and S9, B and C). NusG$\mathrm{C}$ interacts with $\mathrm{S} 10$ in the manner expected from published structures of a complex of NusG and S10 and of a complex of NusG and a ribosome [(2, 9); figs. S3G and S4H). EM density maps show unambiguous density for NusG-N, NusG-C, and most residues of the NusG linker (Figs. 3D and 4D and figs. S3G and S4H), and, at lower contour levels, show density for all residues of the NusG linker (Figs. $3 \mathrm{C}$ and $4 \mathrm{C}$ ). Corresponding EM maps obtained in the absence of NusG do not show TTC-B (fig. S8), indicating that NusG bridging is functionally important for the formation and/or the stability of TTC-B. The NusG bridging hypothesized in refs. (2) and (9) is unequivocally verified.

We first obtained structures of TTC-B in the presence of NusG and absence of NusA (NusG-TTC-B; Fig. 3, figs. S3 and $\mathrm{S} 4$, and table S1). Molecular modelling indicated that NusGTTC-B potentially could accommodate binding of NusA (fig. S10A). Therefore, we sought, and obtained, corresponding structures of TTC-B in the presence of both NusG and NusA (NusA-NusG-TTC-B; Fig. 4, fig. S5, and table S1). As compared to structure determination of TTC-B in the absence of NusA, structure determination of TTC-B in the presence of NusA was associated with substantially higher particle populations ( $4 \%$ vs. $45 \%$ for $\mathrm{n}=8,18 \%$ vs. $28 \%$ for $\mathrm{n}=9$, and $17 \%$ vs. $40 \%$ for $\mathrm{n}=10)$ and substantially higher resolutions (12.6 $\AA$ vs. 3.1 $\AA$ for $\mathrm{n}=8,4.7 \AA$ vs. $4.2 \AA$ for $\mathrm{n}=9$, and $5.0 \AA$ vs. $3.7 \AA$ for $\mathrm{n}$ $=10$ ), indicating that NusA functionally stabilizes TTC-B. Three NusA-NusG-TTC-B subclasses were obtained: TTC-B1, TTC-B2, and TTC-B3, differing by up to $15^{\circ}$ rotation of RNAP relative to NusA and ribosome (figs. S5 and S7, A and B, and movie S2).

In all NusA-NusG-TTC-B subclasses, RNAP and NusG interact with the ribosome $30 \mathrm{~S}$ head, with RNAP $\beta^{\prime}$ ZBD contacting ribosomal protein $\mathrm{S} 3$ and NusG contacting ribosomal protein S10 (Fig. 4, C to E, and fig. S6), essentially as in the absence of NusA (Fig. 3, C and D).

In all NusA-NusG-TTC-B subclasses, NusA makes identical-and extensive-interactions with the surface of the ribosome S30 body, involving contacts between NusA KH1 domain and ribosomal proteins S2 and S5 (1,755 $\AA^{2}$ buried surface area; Fig. 4, C to E, and fig. S6). The NusA-ribosome interactions observed here show no similarity to the putative NusA-ribosome interactions reported in 24; the orientation of NusA relative to the ribosome differs by $\sim 180^{\circ}$, and the interactions involve a different module of the ribosome $30 \mathrm{~S}$ subunit (body vs. head).

NusA functions in this context as a large- $70 \AA$ x $50 \AA-$ open rectangular frame that connects RNAP to the ribosome 30S body (Fig. 4G and fig. S7C). One side of the NusA 
rectangular frame interacts with the ribosome 30S body, and three corners of the NusA rectangular frame interact with RNAP, contacting the RNA $\alpha^{\mathrm{I}}$ C-terminal domain $\left(\alpha \mathrm{CTD}^{\mathrm{I}}\right)$, the RNA $\alpha^{\mathrm{II}} \mathrm{C}$-terminal domain $\left(\alpha \mathrm{CTD}^{\mathrm{II}}\right)$, and the RNAP $\beta$ flap-tip helix (FTH) (Fig. 4, F and G, and fig. S7C). The NusA rectangular frame contains an internal flexible linkage, the AR1-AR2 linker (light blue circles in Fig. 4G and fig. S7C), and interacts with RNAP through three flexible linked modules: $\alpha \mathrm{CTD}^{\mathrm{I}}$ and $\alpha \mathrm{CTD}^{\mathrm{II}}$, which are connected to the rest of RNAP through long, flexible linkers [(25); lines in Fig. 4G and fig. $\mathrm{S} 7 \mathrm{C}]$, and $\beta \mathrm{FTH}$, which is connected to the rest of RNAP through flexible connectors [(15-18); black circle in Fig. 4G and fig. S7C]. The internal flexibility and flexible connections enable the NusA-RNAP subcomplex to maintain constant contact with the ribosome $30 \mathrm{~S}$ body, despite differences in orientation of RNAP relative to the ribosome 30S body (fig. S7C and movie S2). We refer to the NusA rectangular frame as the "coupling pantograph," analogizing it to an electricrailway coupling pantograph, the open rectangular frame, with internal flexibility and flexible connections, that enables a locomotive to maintain constant contact with a power cable, despite differences in orientation of the locomotive relative to the cable [(26); fig. S7C and movie S2].

The separation between the RNAP RNA-exit channel and the ribosome mRNA entry portal in TTC-B, together with the open character of the NusA rectangular frame ("coupling pantograph") in TTC-B, provides largely unrestricted access for transcriptional-regulatory factors to bind, and transcriptional-regulatory RNA secondary structures to form, at and adjacent to the mouth of the RNAP RNA-exit channel (fig. S10). Molecular modelling indicates that TTC-B, unlike TTC$\mathrm{A}$, can accommodate formation of the $21 \mathrm{Q}$ antitermination complex $[(16,17)$; fig. S10B) and can accommodate formation of pause and termination RNA hairpins $[(15,18,19)$; fig. S10, $\mathrm{C}$ and D]. In NusA-NusG-TTC-B, positively charged residues of NusA N and S1 domains are positioned to make favorable electrostatic interactions with the hairpin loop of a pause or termination RNA hairpin, and thereby potentially to nucleate formation of a pause or termination RNA hairpin [(15); fig. S7D]. The different orientations of NusA N and S1 domains in NusA-NusG-TTC-B subclasses B1, B2, and B3 possibly enable interactions with different-length pause and termination RNA hairpins, with B1 accommodating shorter hairpins and B2 and B3 accommodating longer hairpins (fig. S7D).

Molecular modelling also indicates that TTC-B, unlike TTC-A, is compatible with ribosome 30S-head swivelling, the rotation of the $30 \mathrm{~S}$ head relative to the $30 \mathrm{~S}$ body that occurs during ribosome translocation [(20-22]; fig. S11, B and C, and movies S4 and S5). In NusG-TTC-B, all RNAP-ribosome and NusG-ribosome interactions involve the ribosome $30 \mathrm{~S}$ head; accordingly, 30S-head swivelling can be accommodated by rotation of RNAP and NusG with the 30 S head (fig. S11B, center) and/or by separate rotation of flexibly connected RNAP $\beta^{\prime}$ ZBD and flexibly connected NusG-C with the 30 S head (fig. S11B, right, and movie S4). In NusA-NusG-TTC-B, NusA-ribosome interactions involve the ribosome $30 \mathrm{~S}$ body, and RNAPribosome and NusG-ribosome interactions involve the ribosome $30 \mathrm{~S}$ head; nevertheless-exploiting the internal flexibility and flexible connections of the NusA-RNAP "coupling pantograph"-30S-head swivelling can be accommodated by rotation of RNAP and NusG with the 30 S head (fig. S11B, center) and/or by separate rotation of flexibly connected RNAP $\beta^{\prime}$ ZBD and flexibly connected NusG-C with the $30 \mathrm{~S}$ head (fig. S11B, right, and movie S5).

Based on the observation that TTC-B is compatible with NusG bridging, NusA binding, known functional aspects of transcription, and known functional aspects of translation, we propose that TTC-B modulates NusG-dependent, NusAdependent transcription-translation coupling in E. coli.

The structures presented were determined in the presence of CHAPSO, a non-ionic detergent that has been used extensively in cryo-EM structural analysis of RNAP and RNAP complexes to improve structural homogeneity by disrupting non-specific complexes and weak complexes, and by improving rotational-orientation distributions of particles by reducing interactions with the air-water interface (14-18). Analogous structure determination in the absence of CHAPSO yielded lowresolution maps of TTC-A for nucleic-acid scaffolds with mRNA spacer lengths of 4, 5, 6, and 7 codons (figs. S12 and S13, table S2, and movie S6) and yielded low-resolution maps of two additional complexes, TTC-C and TTC-D, for nucleic-acid scaffolds with mRNA spacer lengths of 7,8 , and 9 codons (figs. S13 to S16, table S2, and movies S7 to S11). The fact that TTC-C and TTC-D are observed only in the absence of CHAPSO suggests TTC-C and TTC-D may involve relatively weak interactions. In TTC-C and TTC-D, interactions between RNAP and ribosome are mediated by RNAP $\beta$ sequence insert 2 [ $\beta$ SI2; also known as $\beta i 9 ;(27,28)$ ], a $60-\AA \AA$ long $\alpha$-helical antiparallel coiled-coil flexibly tethered to the rest of RNAP, and the main interaction is an electrostatic interaction between the tip of $\beta$ SI2 and the ribosome $30 \mathrm{~S}$ subunit (figs. S15 and S16). In TTC-C the orientation of RNAP relative to the ribosome is compatible with NusG bridging (fig. S15), and in TTC-D the orientation of RNAP relative to the ribosome is incompatible with NusG bridging (fig. S16). The structures suggest that TTC-C and TTC-D could play roles in NusG-dependent transcription-translation coupling and in NusG-independent transcription-translation coupling, respectively. The structural module that mediates RNAP-ribosome interaction in TTC-C and TTC-D-BSI2-is not essential for growth in rich media (26), but is essential for growth in minimal media (26), implying that TTC-C and TTC-D are unlikely to be important for transcriptiontranslation coupling in general, but may be important for transcription-translation coupling in specific transcription units in specific regulatory contexts (6). Further analysis will be needed 
to determine whether, and, if so, in which contexts, TTC-C and TTC-D function in transcription-translation coupling in E. coli.

The results presented define four structural classes of TTCsTTC-A [the previously reported "expressome"; (12)], TTC-B, TTC$\mathrm{C}$, and TTC-D-and show that TTC-B has structural properties indicating it mediates NusG-dependent, NusA-dependent transcription-translation coupling in E. coli.

The results presented reframe our understanding of the structural and mechanistic basis of transcription-translation coupling. The results provide high-resolution structures of the previously described "expressome" [(12); TTC-A] that demonstrate the incompatibility of the previously described "expressome" with general transcription-translation coupling. In addition, the results provide high-resolution structures of a new structural state, TTC-B, with properties assignable to general, NusG-dependent, NusA-dependent transcription-translation coupling, show that NusG stabilizes TTC-B by bridging RNAP and the ribosome $30 \mathrm{~S}$ head, show that NusA stabilizes TTC-B by bridging RNAP and the ribosome 30S body, and show that NusA serves as a "coupling pantograph" that bridges RNAP and the ribosome $30 \mathrm{~S}$ body in a flexible manner that allows rotation of RNAP relative to the ribosome $30 \mathrm{~S}$ body. Finally, the results provide testable new hypotheses regarding the identities of the RNAP and NusA structural modules crucial for transcriptiontranslation coupling (RNAP $\beta^{\prime}$ ZBD and NusA KH1) and regarding the interactions made by those structural modules (interactions with ribosomal protein S3 in the S30 head and interactions with ribosomal proteins $\mathrm{S} 2$ and $\mathrm{S} 5$ in the S30 body).

\section{REFERENCES AND NOTES}

1. O. L. Miller Jr., B. A. Hamkalo, C. A. Thomas Jr., Visualization of bacterial genes in action. Science 169, 392-395 (1970). doi:10.1126/science.169.3943.392 Medline

2. B. M. Burmann, K. Schweimer, X. Luo, M. C. Wahl, B. L. Stitt, M. E. Gottesman, P. Rösch, A NusE:NusG complex links transcription and translation. Science 328, 501-504 (2010). doi:10.1126/science.1184953 Medline

3. S. Proshkin, A. R. Rahmouni, A. Mironov, E. Nudler, Cooperation between translating ribosomes and RNA polymerase in transcription elongation. Science 328, 504508 (2010). doi:10.1126/science.1184939 Medline

4. D. Castro-Roa, N. Zenkin, In vitro experimental system for analysis of transcriptiontranslation coupling. Nucleic Acids Res. 40, e45 (2012). doi:10.1093/nar/gkr1262 Medline

5. K. McGary, E. Nudler, RNA polymerase and the ribosome: The close relationship. Curr. Opin. Microbiol. 16, 112-117 (2013). doi:10.1016/i.mib.2013.01.010 Medline

6. I. Artsimovitch, Rebuilding the bridge between transcription and translation. Mol. Microbiol. 108, 467-472 (2018). doi:10.1111/mmi.13964 Medline

7. S. Saxena, K. K. Myka, R. Washburn, N. Costantino, D. L. Court, M. E. Gottesman, Escherichia colitranscription factor NusG binds to 70 S ribosomes. Mol. Microbiol. 108, 495-504 (2018). doi:10.1111/mmi.13953 Medline

8. F. Stevenson-Jones, J. Woodgate, D. Castro-Roa, N. Zenkin, Ribosome reactivates transcription by physically pushing RNA polymerase out of transcription arrest. Proc. Natl. Acad. Sci. U.S.A. 117, 8462-8467 (2020). doi:10.1073/pnas.1919985117 Medline

9. R. Washburn, P. Zuber, M. Sun, Y. Hashem, B. Shen, W. Li, S. Harvey, S. Knauer, J. Frank, M. Gottesman, Escherichia coli NusG links the lead ribosome with the transcription elongation complex. bioRxiv 871962 [Preprint]. 11 December 2019; https://doi.org/10.1101/871962.

10. M. Chen, K. Fredrick, Measures of single- versus multiple-round translation argue against a mechanism to ensure coupling of transcription and translation. Proc. Natl. Acad. Sci. U.S.A. 115, 10774-10779 (2018). doi:10.1073/pnas.1812940115 Medline
11. M. Strauß, C. Vitiello, K. Schweimer, M. Gottesman, P. Rösch, S. H. Knauer, Transcription is regulated by NusA:NusG interaction. Nucleic Acids Res. 44, 59715982 (2016). doi:10.1093/nar/gkw423 Medline

12. R. Kohler, R. A. Mooney, D. J. Mills, R. Landick, P. Cramer, Architecture of a transcribing-translating expressome. Science 356, 194-197 (2017). doi:10.1126/science.aal3059 Medline

13. G. Demo, A. Rasouly, N. Vasilyev, V. Svetlov, A. B. Loveland, R. Diaz-Avalos, N. Grigorieff, E. Nudler, A. A. Korostelev, Structure of RNA polymerase bound to ribosomal 30S subunit. el ife 6, e28560 (2017). doi:10.7554/eLife.28560 Medline

14. J. Y. Kang, R. A. Mooney, Y. Nedialkov, J. Saba, T. V. Mishanina, I. Artsimovitch, R. Landick, S. A. Darst, Structural basis for transcript elongation control by NusG family universal regulators. Cell 173, 1650-1662.e14 (2018). doi:10.1016/i.cell.2018.05.017 Medline

15. X. Guo, A. G. Myasnikov, J. Chen, C. Crucifix, G. Papai, M. Takacs, P. Schultz, A. Weixlbaumer, Structural basis for NusA stabilized transcriptional pausing. Mol. Cell 69, 816-827.e4 (2018). doi:10.1016/i.molcel.2018.02.008 Medline

16. J. Shi, X. Gao, T. Tian, Z. Yu, B. Gao, A. Wen, L. You, S. Chang, X. Zhang, Y. Zhang, $Y$. Feng, Structural basis of Q-dependent transcription antitermination. Nat Commun. 10, 2925 (2019). doi:10.1038/s41467-019-10958-8 Medline

17. Z. Yin, J. T. Kaelber, R. H. Ebright, Structural basis of Q-dependent antitermination. Proc. Natl. Acad. Sci. U.S.A. 116, 18384-18390 (2019). doi:10.1073/pnas.1909801116 Medline

18. J. Y. Kang, T. V. Mishanina, M. J. Bellecourt, R. A. Mooney, S. A. Darst, R. Landick, RNA polymerase accommodates a pause RNA hairpin by global conformational rearrangements that prolong pausing. Mol. Cell 69, 802-815.e5 (2018). do:10.1016/imolcel.2018.01.018 Medline

19. J. W. Roberts, Mechanisms of bacterial transcription termination. J. Mol. Biol. 431 4030-4039 (2019). doi:10.1016/i.jmb.2019.04.003 Medline

20. B. S. Schuwirth, M. A. Borovinskaya, C. W. Hau, W. Zhang, A. Vila-Sanjurjo, J. M. Holton, J. H. D. Cate, Structures of the bacterial ribosome at 3.5 A resolution. Science 310, 827-834 (2005). doi:10.1126/science.1117230 Medline

21. A. Ratje, J. Loerke, A. Mikolajka, M. Brünner, P. Hildebrand, A. Starosta, A. Dönhöfer, S. Connell, P. Fucini, T. Mielke, P. Whitford, J. Onuchic, Y. Yu, K. Sanbonmatsu, R. Hartmann, P. Penczek, D. Wilson, C. Spahn, Head swivel on the ribosome facilitates translocation by means of intra-subunit tRNA hybrid sites. Nature 486, 714-716 (2010)

22. Z. Guo, H. F. Noller, Rotation of the head of the 30 S ribosomal subunit during mRNA translocation. Proc. Natl. Acad. Sci. U.S.A. 109, 20391-20394 (2012) doi:10.1073/pnas.1218999109 Medline

23. M. Webster, M. Takacs, C. Zhu, V. Vidmar, A. Eduljee, M. Abdelkareem, A. Weixlbaumer, Structural basis of transcription-translation coupling and collision in bacteria. bioRxiv 971028 [Preprint]. 2 March 2020; https://doi.org/10.1101/2020.03.01.971028.

24. F. O'Reilly, L. Xue, A. Graziadei, L. Sinn, S. Lenz, D. Tegunov, C. Blötz, W. Hagen, P. Cramer, J. Stülke, J. Mahamid, J. Rappsilber, In-cell architecture of an actively transcribing-translating expressome. bioRxiv 970111 [Preprint]. 28 February 2020; https://doi.org/10.1101/2020.02.28.970111.

25. E. E. Blatter, W. Ross, H. Tang, R. L. Gourse, R. H. Ebright, Domain organization of RNA polymerase $\alpha$ subunit: C-terminal 85 amino acids constitute a domain capable of dimerization and DNA binding. Cell 78, 889-896 (1994). doi:10.1016/S0092-8674(94)90682-3 Medline

26. Wikipedia, "Pantograph (transport)"; https://en.wikipedia.org/wiki/Pantograph_(transport)

27. I. Artsimovitch, V. Svetlov, K. S. Murakami, R. Landick, Co-overexpression of Escherichia coli RNA polymerase subunits allows isolation and analysis of mutant enzymes lacking lineage-specific sequence insertions. J. Biol. Chem. 278, 1234412355 (2003). doi:10.1074/jbc.M211214200 Medline

28. W. J. Lane, S. A. Darst, Molecular evolution of multisubunit RNA polymerases: Sequence analysis. J. Mol. Biol. 395, 671-685 (2010). doi:10.1016/i.jmb.2009.10.062 Medline

29. G. A. Belogurov, M. N. Vassylyeva, V. Svetlov, S. Klyuyev, N. V. Grishin, D. G. Vassylyev, I. Artsimovitch, Structural basis for converting a general transcription factor into an operon-specific virulence regulator. Mol. Cell 26, 117-129 (2007). Medline

30. V. Molodtsov, E. Sineva, L. Zhang, X. Huang, M. Cashel, S. E. Ades, K. S. Murakami, 
Allosteric effector ppGpp potentiates the inhibition of transcript initiation by DksA. Mol. Cell 69, 828-839.e5 (2018). doi:10.1016/i.molcel.2018.01.035 Medline

31. K. Li, T. Jiang, B. Yu, L. Wang, C. Gao, C. Ma, P. Xu, Y. Ma, Escherichia coli transcription termination factor NusA: Heat-induced oligomerization and chaperone activity. Sci. Rep. 3, 2347 (2013). doi:10.1038/srep02347 Medline

32. I. Artsimovitch, R. Landick, Pausing by bacterial RNA polymerase is mediated by mechanistically distinct classes of signals. Proc. Natl. Acad. Sci. U.S.A. 97, 70907095 (2000). doi:10.1073/pnas.97.13.7090 Medline

33. J. Ederth, C. S. Mandava, S. Dasgupta, S. Sanyal, A single-step method for purification of active His-tagged ribosomes from a genetically engineered Escherichia coli. Nucleic Acids Res. 37, e15 (2009). Medline

34. H. Fan, A. B. Conn, P. B. Williams, S. Diggs, J. Hahm, H. B. Gamper Jr., Y. M. Hou, S. E. O'Leary, Y. Wang, G. M. Blaha, Transcription-translation coupling: Direct interactions of RNA polymerase with ribosomes and ribosomal subunits. Nucleic Acids Res. 45, 11043-11055 (2017). doi:10.1093/nar/gkx719 Medline

35. G. Blaha, U. Stelzl, C. M. Spahn, R. K. Agrawal, J. Frank, K. H. Nierhaus, Preparation of functional ribosomal complexes and effect of buffer conditions on tRNA positions observed by cryoelectron microscopy. Methods Enzymol. 317, 292-309 (2000). doi:10.1016/S0076-6879(00)17021-1 Medline

36. J. M. Robertson, H. Paulsen, W. Wintermeyer, Pre-steady-state kinetic studies on ribosomal translocation. Methods Enzymol. 164, 581-597 (1988). doi:10.1016/S0076-6879(88)64071-7 Medline

37. S. Q. Zheng, E. Palovcak, J. P. Armache, K. A. Verba, Y. Cheng, D. A. Agard, MotionCor2: Anisotropic correction of beam-induced motion for improved cryoelectron microscopy. Nat. Methods 14, 331-332 (2017). doi:10.1038/nmeth.4193 Medline

38. A. Rohou, N. Grigorieff, CTFFIND4: Fast and accurate defocus estimation from electron micrographs. J. Struct. Biol. 192, 216-221 (2015). doi:10.1016/i.jsb.2015.08.008 Medline

39. J. Zivanov, T. Nakane, B. O. Forsberg, D. Kimanius, W. J. Hagen, E. Lindahl, S. H. Scheres, New tools for automated high-resolution cryo-EM structure determination in RELION-3. eLife 7, e42166 (2018). doi:10.7554/eLife.42166 Medline

40. B. Beckert, M. Turk, A. Czech, O. Berninghausen, R. Beckmann, Z. Ignatova, J. M. Plitzko, D. N. Wilson, Structure of a hibernating 100 S ribosome reveals an inactive conformation of the ribosomal protein S1. Nat. Microbiol. 3, 1115-1121 (2018). do::10.1038/s41564-018-0237-0 Medline

41. P. Tian, A. Steward, R. Kudva, T. Su, P. J. Shilling, A. A. Nickson, J. J. Hollins, R. Beckmann, G. von Heijne, J. Clarke, R. B. Best, Folding pathway of an Ig domain is conserved on and off the ribosome. Proc. Natl. Acad. Sci. U.S.A. 115, E11284E11293 (2018). doi:10.1073/pnas.1810523115 Medline

42. L. B. Jenner, N. Demeshkina, G. Yusupova, M. Yusupov, Structural aspects of messenger RNA reading frame maintenance by the ribosome. Nat. Struct. Mol. Biol. 17, 555-560 (2010). doi:10.1038/nsmb.1790 Medline

43. E. F. Pettersen, T. D. Goddard, C. C. Huang, G. S. Couch, D. M. Greenblatt, E. C. Meng, T. E. Ferrin, UCSF Chimera-A visualization system for exploratory research and analysis. J. Comput. Chem. 25, 1605-1612 (2004). doi:10.1002/icc.20084 Medline

44. D. Liebschner, P. Afonine, M. Baker, G. Bunkoczi, V. Chen, T. Croll, B. Hintze, L. Hung, S. Jain, A. McCoy, N. Moriarty, R. Oeffner, B. Poon, M. Prisant, R. J. Read, J. S. Richardson, D. C. Richardson, M. D. Sammito, O. V. Sobolev, D. H. Stockwell, T. C. Terwilliger, A. G. Urzhumtsev, L. L. Videau, C. J. Williams, P. D. Adams, R. J. Read, J. Richardson, D. Richardson, M. Sammito, O. Sobolev, D. Stockwell, T. Terwilliger, A. Urzhumtsev, L. Videau, C. Williams, P. Adams, Macromolecular structure determination using $X$-rays, neutrons and electrons: Recent developments in Phenix. Acta Crystallogr. D Biol. Crystallogr. 75, 861-877 (2019). doi:10.1107/S2059798319011471

45. P. Emsley, B. Lohkamp, W. G. Scott, K. Cowtan, Features and development of Coot. Acta Crystallogr. D Biol. Crystallogr. 66, 486-501 (2010). doi:10.1107/S0907444910007493 Medline

46. C. Suloway, J. Pulokas, D. Fellmann, A. Cheng, F. Guerra, J. Quispe, S. Stagg, C. S. Potter, B. Carragher, Automated molecular microscopy: The new Leginon system. J. Struct. Biol. 151, 41-60 (2005). doi:10.1016/i.jsb.2005.03.010 Medline

47. D. Mastronarde, Advanced data acquisition from electron microscopes with
SerialEM. Microsc. Microanal. 24(S1), 864-865 (2018). doi:10.1017/S1431927618004816

48. A. Cheng, E. T. Eng, L. Alink, W. J. Rice, K. D. Jordan, L. Y. Kim, C. S. Potter, B. Carragher, High resolution single particle cryo-electron microscopy using beamimage shift. J. Struct. Biol. 204, 270-275 (2018). doi:10.1016/j.jsb.2018.07.015 Medline

49. M. Su, goCTF: Geometrically optimized CTF determination for single-particle cryoEM. J. Struct. Biol. 205, 22-29 (2019). doi:10.1016/j.jsb.2018.11.012 Medline

50. M. Selmer, C. M. Dunham, F. V. Murphy 4th, A. Weixlbaumer, S. Petry, A. C. Kelley, J. R. Weir, V. Ramakrishnan, Structure of the 70 S ribosome complexed with mRNA and tRNA. Science 313, 1935-1942 (2006). doi:10.1126/science.1131127Medline

51. J. Zhou, L. Lancaster, J. P. Donohue, H. F. Noller, How the ribosome hands the Asite tRNA to the P site during EF-G-catalyzed translocation. Science 345, 11881191 (2014). doi:10.1126/science.1255030 Medline

\section{ACKNOWLEDGMENTS}

We thank the Rutgers University Cryo-EM Core facility, University of Michigan Life Sciences Institute Cryo-EM Facility, National Center for CryoEM Access and Training (supported by NIH grant GM129539, Simons Foundation grant SF349247, and New York state grants), and Pacific Northwest Center for CryoEM (supported by NIH grant GM129547 and Department of Energy Environmental Molecular Sciences Laboratory) for microscope access; K. Kuznedelov and K. Severinov for plasmids; and L. Minakhin, B. Nickels, and J. Winkelman for discussion; and E. Eng and $\mathrm{H}$. Wei for assistance. Funding: This work was supported by University of California discretionary funds to G.B. University of Michigan discretionary funds to M.S., and National Institutes of Health (NIH) grant GM041376 to R.H.E. Author Contributions: V.M. and G.B. prepared biomolecules. C.W., V.M., E.F., J.K., and M.S. collected data. C.W., V.M, J.K., M.S., and R.H.E. analyzed data. C.W., V.M., and R.H.E. prepared figures. R.H.E. designed experiments and wrote the manuscript. Data and Material Availability:Cryo-EM micrographs have been deposited in the Electron Microscopy Public Image Archive Resource (EMPIAR accession codes 10467 and 10468). Cryo-EM maps and atomic models have been deposited in the Electron Microscopy Database (EMDB accession codes 21386, 21468, 21469, 21470. $21471,21472,21474,21475,21476,21477,21482,21483,21485,21486,21494$, 22082, 22084, 22087, 22107, 22141, 22142, 22181, 22192, and 22193) and the Protein Database (PDB accession codes 6VU3, 6VYQ, 6VYR, 6VYS, 6VYT, 6VYU, 6VYW, 6VYX, 6VYY, 6VYZ, 6VZJ, 6VZ2, 6VZ3, 6VZ5, 6VZ7, 6XDQ, 6XDR, 6XGF, $6 \mathrm{XII}, 6 \mathrm{XIJ}, 6 \mathrm{X6 \textrm {T }}, 6 \mathrm{X7F}, 6 \mathrm{X7K}$, and $6 \mathrm{X9Q}$ ). Unique materials are available from the authors on request.

\section{SUPPLEMENTARY MATERIALS}

science.sciencemag.org/cgi/content/full/science.abb5317/DC1

Materials and Methods

Figs. S1 to S16

Tables S1 and S2

References (29-51)

Movies S1 to S11

MDAR Reproducibility Checklist

1 March 2020; accepted 17 July 2020

Published online 20 August 2020

10.1126/science.abb5317 


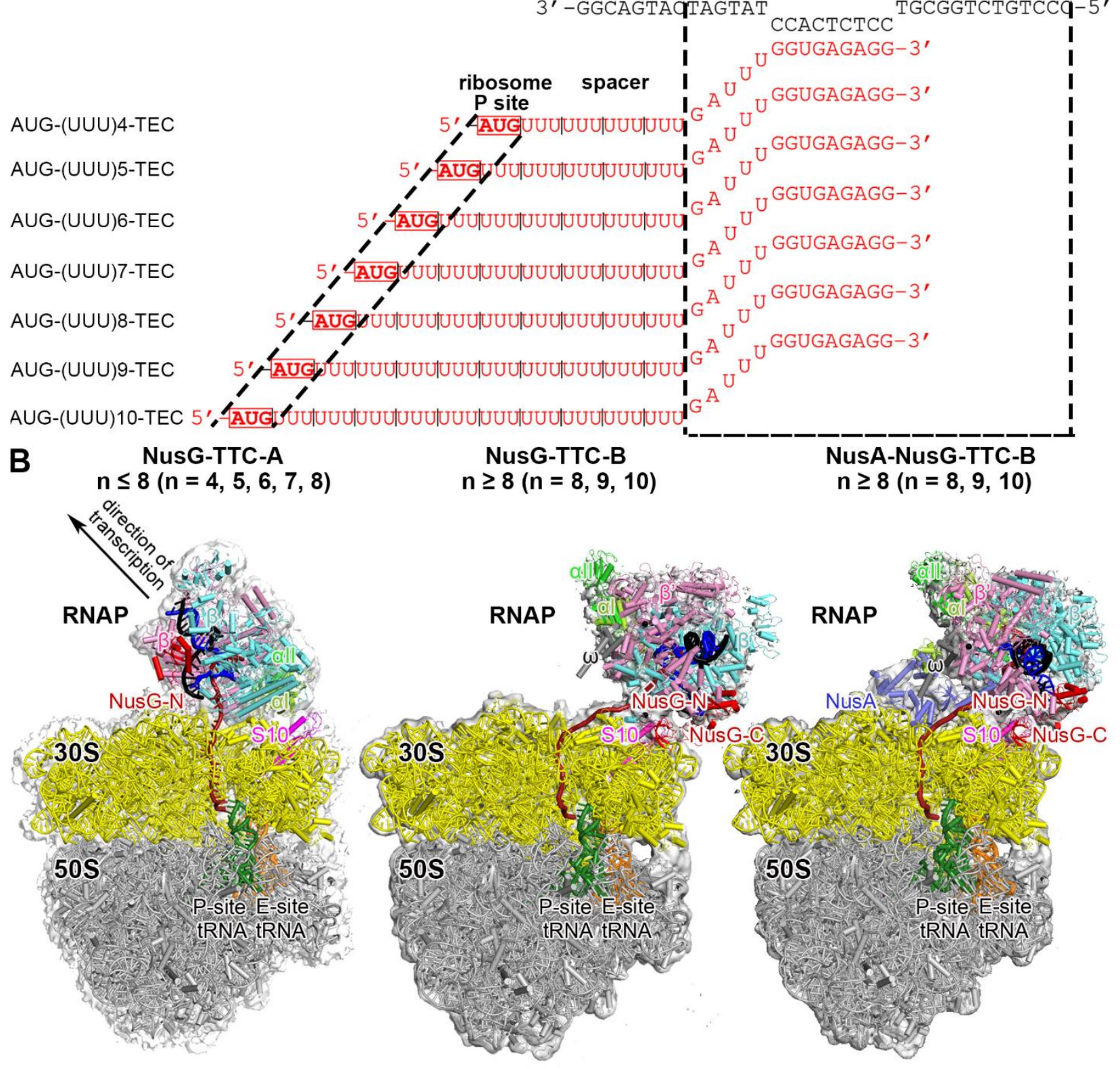

Fig. 1. Structure determination: TTCs. (A) Nucleic-acid scaffolds. Each scaffold comprises nontemplate- and template-strand oligodeoxyribonucleotides (black) and one of seven oligoribonucleotides having spacer length $\mathrm{n}$ of 4, 5, 6, 7, 8, 9, or 10 codons (red), corresponding to mRNA. Dashed black box labeled "TEC," portion of nucleic-acid scaffold that forms TEC upon addition of RNAP (10 nt nontemplate- and template-strand ssDNA segments forming "transcription bubble," 10 nt of mRNA engaged with template-strand DNA as RNA-DNA "hybrid," and 5 nt of mRNA, on diagonal, in RNAP RNA-exit channel); dashed black lines labeled "ribosome P-site," mRNA AUG codon intended to occupy ribosome active-center $P$ site upon addition of ribosome and tRNA ${ }^{\text {fMet; }}$ " spacer," mRNA spacer between TEC and AUG codon in ribosome active-center P site. (B) Cryo-EM structures of NusG-TTC-A (obtained with spacer lengths of 4-8 codons), NusG-TTC-B (obtained with spacer lengths of 8-10 codons), and NusA-NusG-TTC-B (obtained with spacer lengths of 8-10 codons). Structures shown are NusG-TTC-A (3.7 $\AA ; n=4$; table S1), NusGTTC-B (4.7 $\AA ; n=9$; table S1), and NusA-NusG-TTC-B2 (3.5 $\AA$; $n=8$; table S1). Images show EM density (gray surface) and fit (ribbons) for TEC, NusG and NusA (at top; direction of transcription, defined by downstream dsDNA, indicated by arrow in left panel and directly toward viewer in center and right panels) and for ribosome 30S and 50S subunits and $\mathrm{P}$ - and E-site tRNAs (at bottom). RNAP $\beta^{\prime}, \beta, \alpha^{\prime}, \alpha^{\prime \prime}$, and $\omega$ subunits are in pink, cyan, light green, and dark green, and gray; 30S subunit, $50 S$ subunit, P-site tRNA, E-site tRNA are in yellow, gray, green, and orange; DNA nontemplate strand, DNA template strand, and mRNA are in black, blue, and brick-red (brick-red dashed line where modeled). NusG, NusA, and ribosomal protein S10 are in red, light blue, and magenta. Ribosome L7/L12 stalk omitted for clarity in this and all subsequent images. 

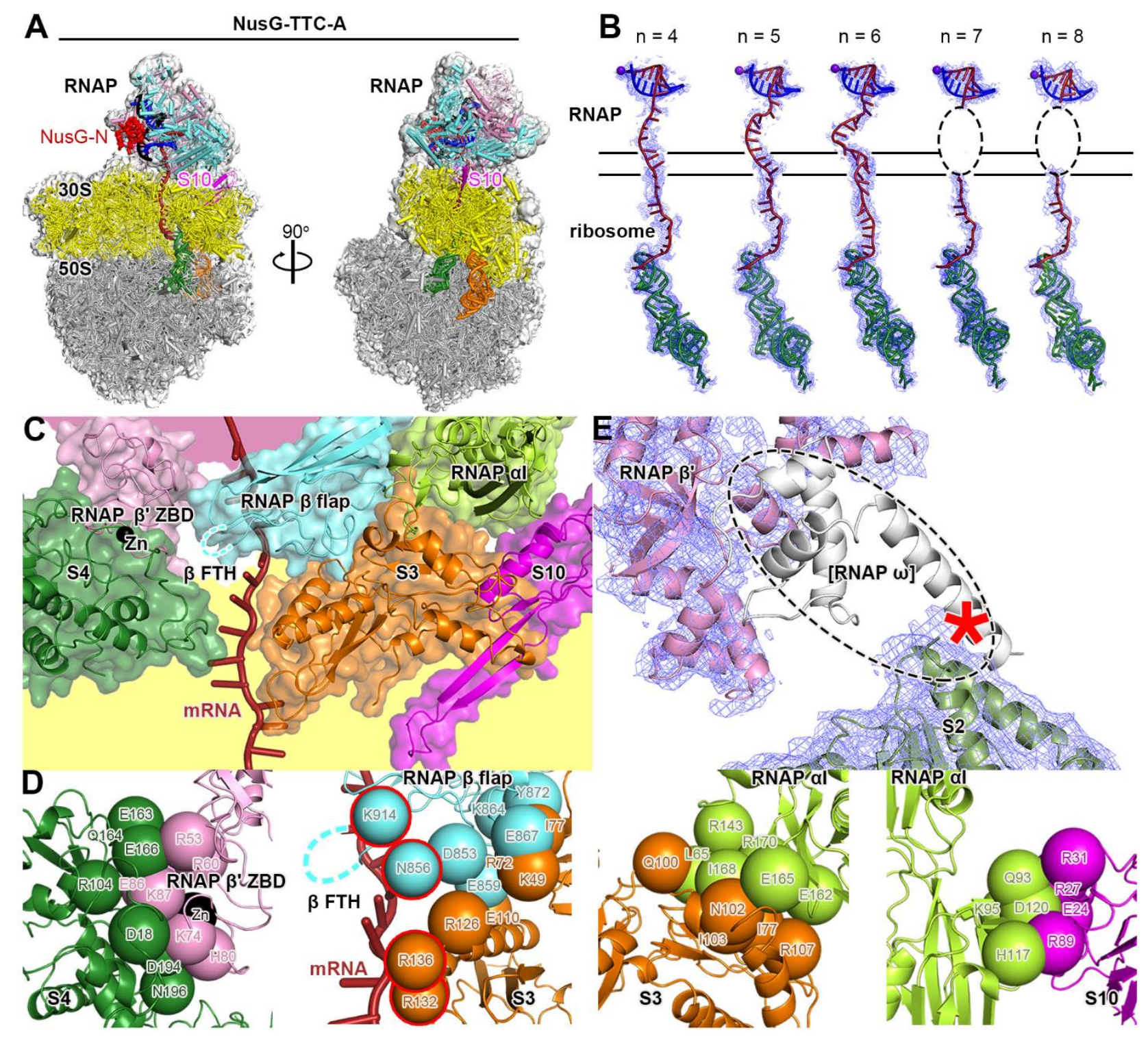

Fig. 2. Cryo-EM structure of NusG-TTC-A. (A) Structure of NusG-TTC-A (3.7 $\AA ; n=4$; table S1). Two orthogonal views. Colors as in Fig. 1B. (B) Accommodation of mRNA spacer lengths of 4, 5, 6, 7 and 8 codons in NusG-TTC-A. EM density, blue mesh; mRNA, brick-red (disordered mRNA nucleotides indicated by dashed oval); template-strand DNA in RNA-DNA hybrid, blue; RNAP active-center catalytic $\mathrm{Mg}^{2+}$, purple sphere; tRNA in ribosome $\mathrm{P}$ site, green. Upper and lower black horizontal lines indicate edges of RNAP and ribosome. (C) RNAP-ribosome interface in NusGTTC-A ( $n=4$; identical interface for $n=5,6$, 7, or 8 ), showing RNAP $\beta$ ' zinc binding domain, (ZBD, pink; $Z n^{2+}$ ion as black sphere), RNAP $\beta$ flap, cyan, RNAP $\beta$ flap tip helix ( $\beta$ FTH; disordered residues indicated by cyan dashed line), and RNAP $\alpha^{\prime}$ (green) interacting with ribosomal proteins S4 (forest green), S3 (orange), and S10 (magenta) and with mRNA (brick red). Portions of RNAP $\beta^{\prime}$ and ribosome $30 S$ not involved in interactions are shaded pink and yellow, respectively. (D) RNAP-ribosome interactions involving RNAP $\beta^{\prime}$ ZBD and S4 (subpanel 1), RNAP $\beta$ flap and S3 (subpanel 2; $\beta \mathrm{FTH}$, dashed line; $\beta$ and $\mathrm{S} 3$ residues that interact with $\mathrm{mRNA}$, cyan and orange spheres with red outlines; mRNA, brick-red), RNAP $\alpha^{\prime}$ and S3 (subpanel 3), and RNAP $\alpha^{\prime}$ and S10 (subpanel 4). Other colors as in (C). (E) Absence of EM density for RNAP $\omega$ subunit. EM density, blue mesh; atomic models for RNAP $\beta^{\prime}$ and S2, pink ribbon and forest-green ribbon, respectively; location of missing EM density for $\omega$, dashed oval; $\omega$ in TEC in absence of ribosome [PDB 6P19; (17)], white ribbon. 

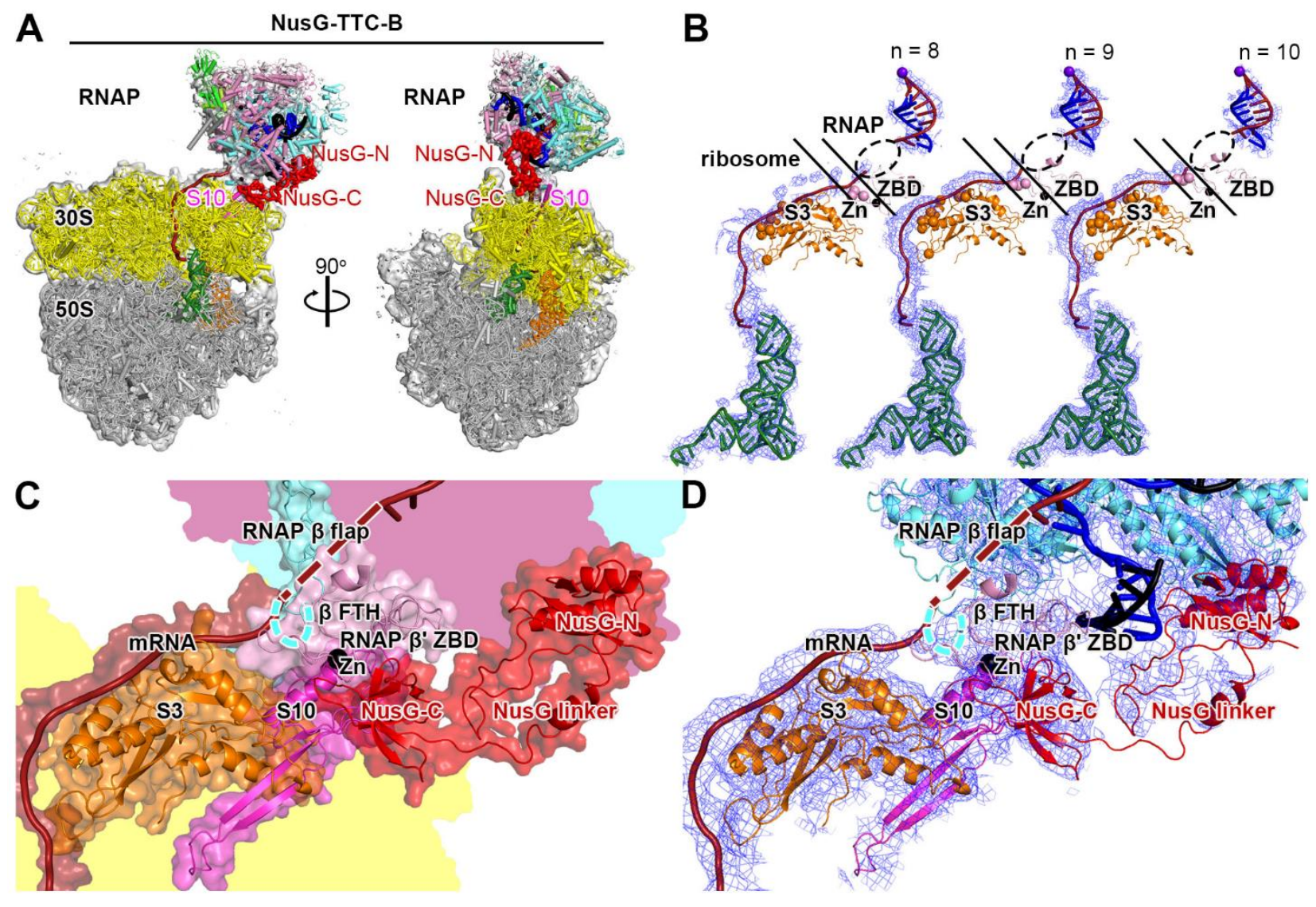

Fig. 3. Cryo-EM structure of NusG-TTC-B. (A) Structure of NusG-TTC-B (4.7 $\AA ; n=9$; table S1). Views and colors as in Fig. 2A. (B) Accommodation of mRNA spacer lengths of 8, 9, and 10 codons in NusG-TTC-B. EM density, blue mesh; mRNA, brick-red (disordered mRNA nucleotides indicated by dashed oval); template-strand DNA in RNA-DNA hybrid, blue; RNAP active-center catalytic $\mathrm{Mg}^{2+}$, purple sphere; tRNA in ribosome P site, green; ribosomal protein S3, orange (positively charged residues positioned to contact mRNA as orange spheres); RNAP $\beta^{\prime}$ zinc binding domain (ZBD, pink; $\mathrm{Zn}^{2+}$ ion as black sphere; positively charged residues positioned to contact mRNA as pink spheres). Upper and lower black diagonal lines indicate edges of RNAP and ribosome. (C) RNAP-ribosome interface and NusG bridging in NusG-TTC-B ( $n=9$; identical interface for $n=8$, 9, or 10). RNAP $\beta$ ' zinc binding domain, (ZBD, pink; $Z n^{2+}$ ion as black sphere) interacts with ribosomal protein S3 (orange) and mRNA (brick red). NusG (red) bridges RNAP and ribosome, with NusG-N interacting with RNAP and NusG-C interacting with ribosomal protein S10 (magenta). Portions of RNAP $\beta^{\prime}, \beta$, and ribosome 30 S not involved in interactions are shaded pink, cyan, and yellow, respectively. (D) As C, showing cryo-EM density as blue mesh. 
A

NusA-NusG-TTC-B
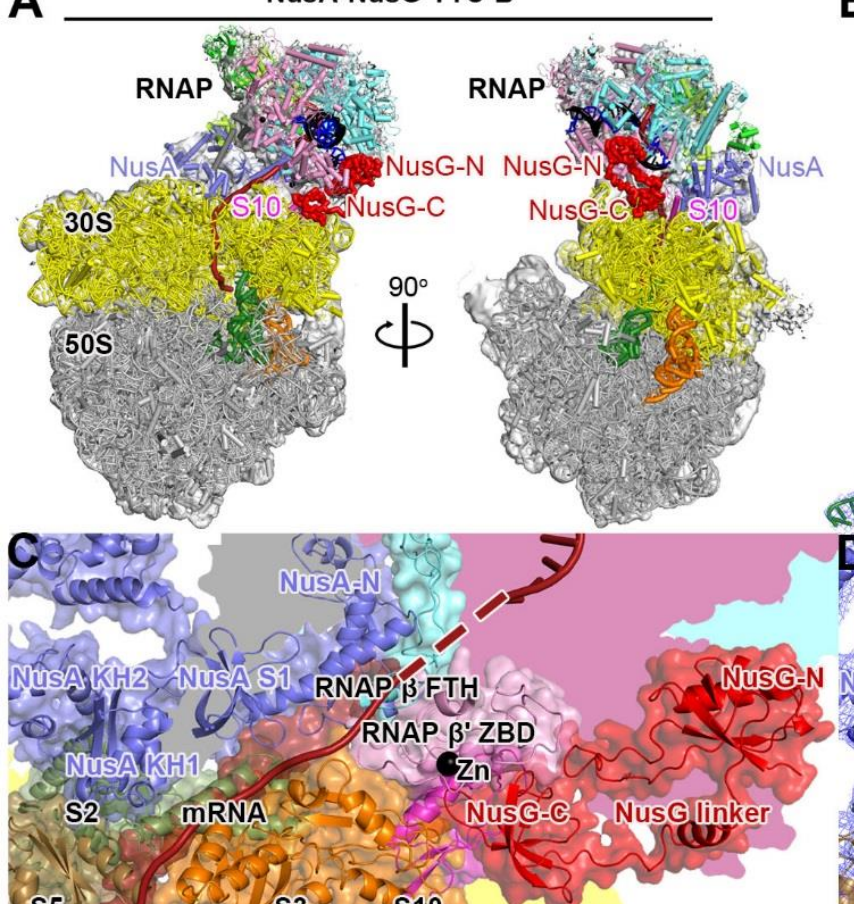

s5 (s) s3

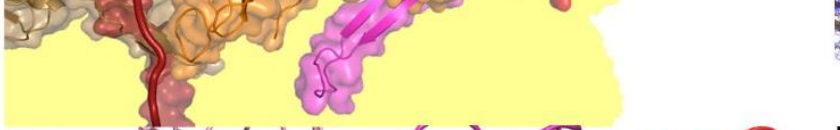

E RNAP $\beta^{\prime} Z Z^{\prime}$

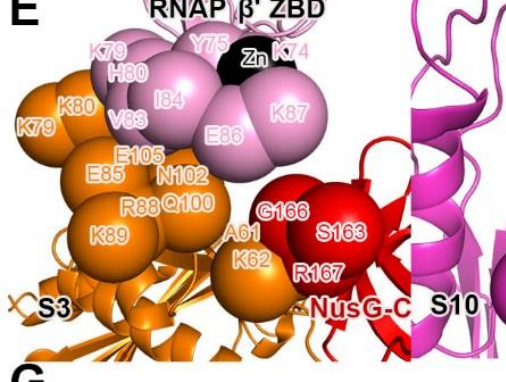

G

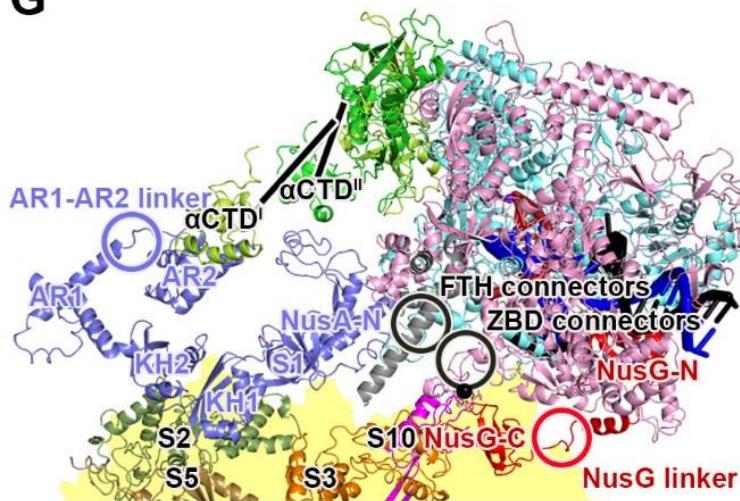

B

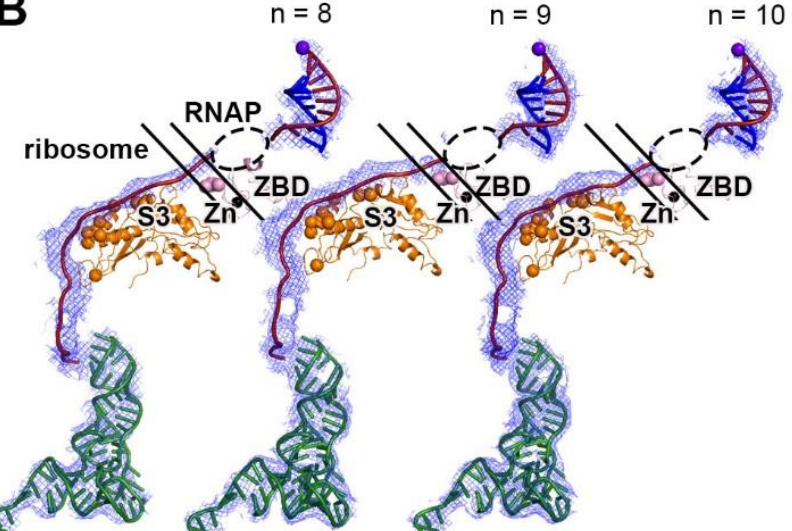

Pe rover
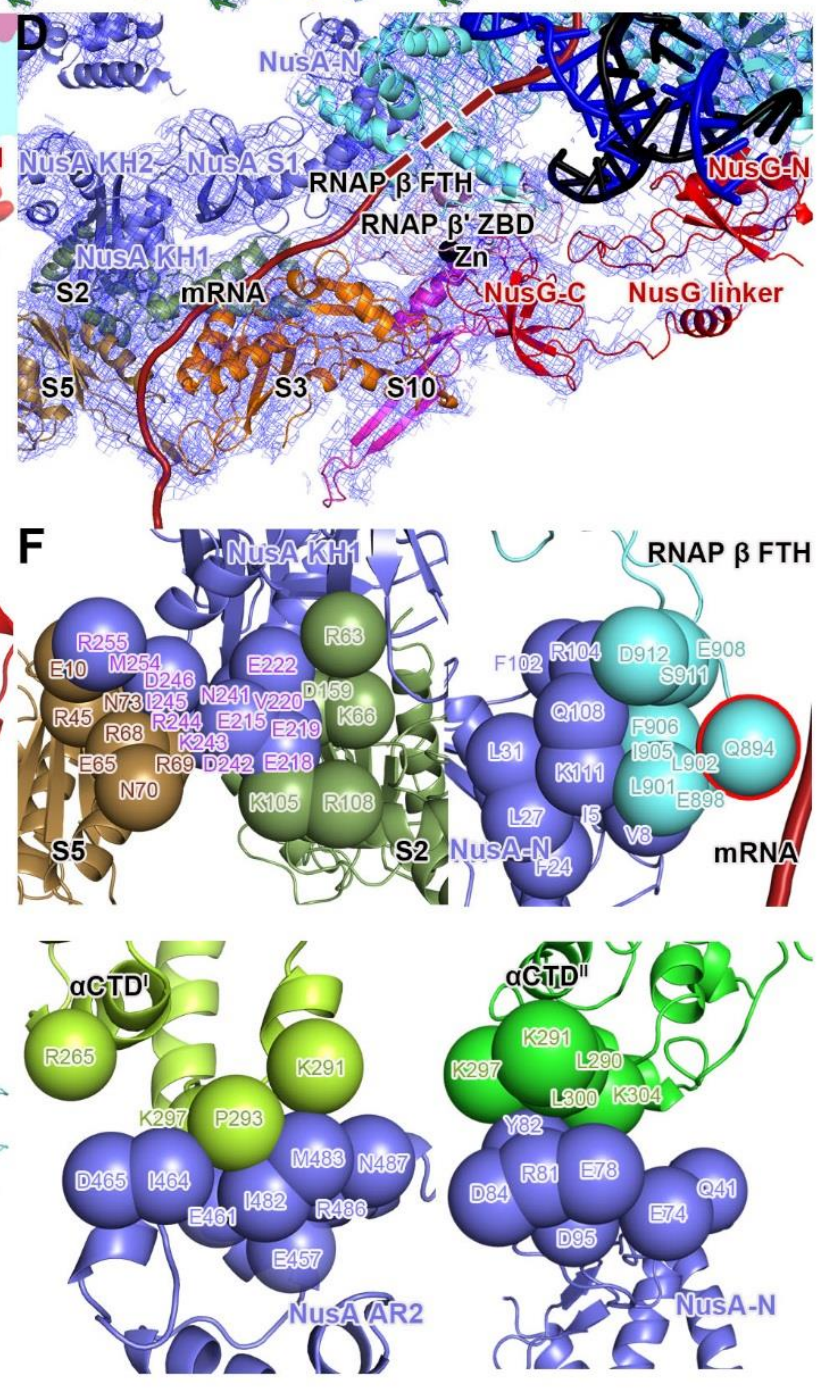
Fig. 4. Cryo-EM structure of NusA-NusG-TTC-B. (A) Structure of NusA-NusG-TTC-B (NusA-NusG-TTC-B2; 3.5 A; $\mathrm{n}=9$; table S1). NusA, light blue. Views and other colors as in Figs. 2A and 3A. (B) Accommodation of mRNA spacer lengths of 8, 9, and 10 codons in NusA-NusG-TTC-B. Views and colors as in Fig. 3B. (C) RNAP-ribosome interface, NusG bridging, and NusA binding in NusA-NusG-TTC-B ( $n=9$; identical interface for $n=8$, 9, or 10$)$. RNAP $\beta^{\prime}$ zinc binding domain, (ZBD, pink; $\mathrm{Zn}^{2+}$ ion as black sphere) interacts with ribosomal protein $\mathrm{S} 3$ (orange) and mRNA (brick red). NusG (red) bridges RNAP and ribosome, with NusG-N interacting with RNAP and NusG-C interacting with ribosomal protein S10 (magenta). NusA (light blue) KH1 domain interacts with ribosomal proteins S5 and S2 (brown and forest green). Portions of RNAP $\beta^{\prime}, \beta, \omega$, and ribosome $30 S$ not involved in interactions are shaded pink, cyan, gray, and yellow, respectively. (D) As C, showing cryo-EM density as blue mesh. (E) RNAP-ribosome interactions involving RNAP $\beta^{\prime}$ ZBD and S3 (subpanel 1) and NusG-ribosome interactions involving NusG-C and S10 (subpanel 2). (F) NusA-ribosome interactions involving NusA KH1 and S5 and S2 (subpanel 1) and NusA-RNAP interactions involving NusA-N and RNAP $\beta$ FTH (subpanel 2; $\beta$ FTH residue that interacts with mRNA, cyan sphere with red outline; mRNA, brick-red), NusA AR2 and RNAP $\alpha$ CTD' (subpanel 3), and NusA-N and RNAP $\alpha$ CTD" (subpanel 4). (G) Points of flexibility in NusA-NusG-TTC-B (NusA "coupling pantograph"): flexible linkage in NusA structure (AR1-AR2 linker; light blue circle), three flexible linkages between NusA and RNAP ( $\alpha$ CTD' linker, $\alpha$ CTD" linker, and $\beta$ FTH connectors; black lines and black circle), flexible linkage between RNAP and ribosome ( $\beta^{\prime}$ ZBD connectors; black circle), and flexible NusG bridging of RNAP and ribosome (NusG linker; red circle). 